\title{
Landmarks or panoramas: what do navigating ants attend to for guidance?
}

\author{
Antoine Wystrach ${ }^{1,2^{*}}$, Guy Beugnon ${ }^{2}$ and Ken Cheng ${ }^{1}$
}

\begin{abstract}
Background: Insects are known to rely on terrestrial landmarks for navigation. Landmarks are used to chart a route or pinpoint a goal. The distant panorama, however, is often thought not to guide navigation directly during a familiar journey, but to act as a contextual cue that primes the correct memory of the landmarks.

Results: We provided Melophorus bagoti ants with a huge artificial landmark located right near the nest entrance to find out whether navigating ants focus on such a prominent visual landmark for homing guidance. When the landmark was displaced by small or large distances, ant routes were affected differently. Certain behaviours appeared inconsistent with the hypothesis that guidance was based on the landmark only. Instead, comparisons of panoramic images recorded on the field, encompassing both landmark and distal panorama, could explain most aspects of the ant behaviours.

Conclusion: Ants navigating along a familiar route do not focus on obvious landmarks or filter out distal panoramic cues, but appear to be guided by cues covering a large area of their panoramic visual field, including both landmarks and distal panorama. Using panoramic views seems an appropriate strategy to cope with the complexity of natural scenes and the poor resolution of insects' eyes. The ability to isolate landmarks from the rest of a scene may be beyond the capacity of animals that do not possess a dedicated object-perception visual stream like primates.
\end{abstract}

Keywords: ant, insect navigation, panoramic views, landmark, route learning

\section{Introduction}

Many insects use terrestrial objects - landmarks - for navigation. Ants and bees in particular are known to rely on landmarks both to pinpoint a goal [1-4], and also to chart routes, which are typically idiosyncratic paths through a landscape dotted with landmarks [5-9]. Knowledge about how insects exploit such landmark information comes mostly from studies conducted in visually controlled and impoverished conditions, like experimental rooms or deserts, where the salience of many potential cues is minimal and only experimental landmarks are made prominent $[1,2,4,10-14]$.

Concurrently, studies conducted in visually rich environments suggested that ants and bees ignore the features of familiar landmarks if they are presented within a wrong panoramic context [15]. This led to the idea

\footnotetext{
* Correspondence: wystrach@cict.fr

'Department of Biological Sciences, Macquarie University, Sydney, NSW 2109 Australia

Full list of author information is available at the end of the article
}

that panoramas and landmarks are different cues that have different functions: a class of theories claims that the panorama serves as a contextual cue that triggers the recall of the appropriate landmark memory, on which guidance is based [16-18]. The segregation between landmark and panorama seems striking in these experimental conditions. This class of theories, however, faces the question of how insects segregate contextual cues and landmarks in natural environments, with complex depth structures. One theoretical proposal is that the amount of motion parallax is used as a depth cue to filter out distant landmarks [19]. Insects are known to use motion parallax as a depth cue [20-25]. But whether insects use such depth information to segregate out landmarks has not been determined empirically.

It has also been suggested that insects may not segregate landmarks from the panorama at all but are guided instead by cues widespread on their panoramic visual field, which encompass both landmarks and panorama

\section{Biomed Central}


[26-32]. Some results support this class of theories. For instance, multiple landmarks $[1,2,33]$ but also landmarks and panorama $[9,34]$ seem to be "bound together" in insect memories. An imitation of the skyline (elevations of surrounding terrestrial objects) is sufficient for orientation in one species of desert ants [26]. In some cases $[14,30]$, the ants proved able to navigate robustly using pretty much plain white walls or curtains. Such performance can be readily explained by the use of panoramic views that encompass the global shape of the arena [35]. Yet it is difficult to create experimental conditions in which the two classes of theories make different predictions. For example, in a previous study, many manipulations on ants' home routes were conducted [9]. But both classes of theories could account equally well for the large body of results.

We here investigate the effect of displacing a prominent landmark within natural surroundings. A huge black landmark was placed immediately behind a nest entrance of Melophorus bagoti ants (Figure 1). Standing in a flat area devoid of proximal trees, the landmark was designed to stick out from the rest of the panorama, and thus to be as easy as possible for an insect to learn, memorise, and extract from the rest of the scenery. We analysed the paths displayed by the ants in response to displacements of the landmark. In parallel, we recorded

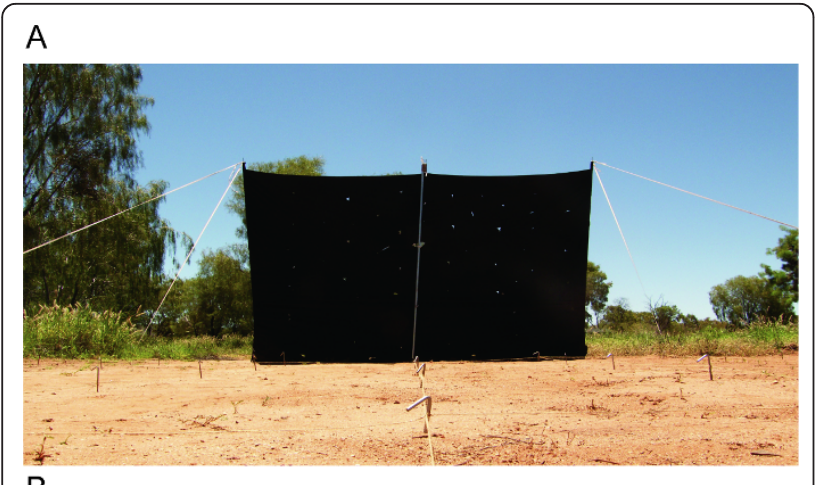

B
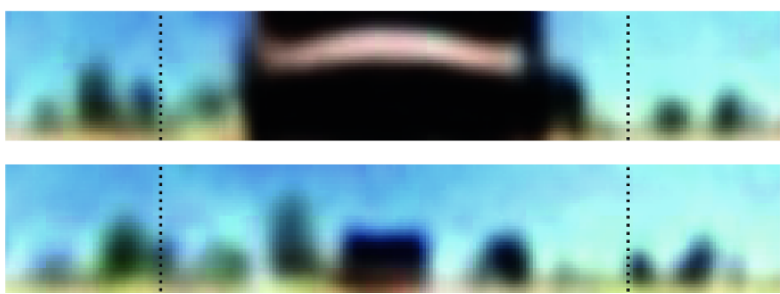

Figure 1 Photos of the experimental set up with the landmark in training position. A. Picture taken $5 \mathrm{~m}$ from the nest. B. Ant'seye picture $\left(300^{\circ}\right.$, resolution of $4^{\circ}$ [49]) taken $5 \mathrm{~m}$ from the nest (bottom) or at the nest position (top). The dashed lines delimit $180^{\circ}$. The landmark was located $90 \mathrm{~cm}$ behind the nest while the closest tree (on the left of the picture A) was located $14 \mathrm{~m}$ away from the nest. The panorama was thus providing very little dynamic change compared to the landmark for ants approaching their nest. panoramic 'ant's eye' images in order to quantify the panoramic alteration of the scenery caused by the landmark displacements. This approach allowed us to relate the ants' behaviour not only to the landmark, but to the whole panoramic scene, providing us with insight on whether navigating ants were focusing on the landmark or using cues widespread on their panoramic visual field.

\section{Results}

Panoramic pictures: image difference distribution

We quantified the alteration of the visual panorama created by the different displacements of the landmark (Figure 2). Across multiple positions, we recorded and compared panoramic images taken with the landmark either in the training position (reference scenery) or displaced (test scenery) (see Additional file 1). The panoramic image difference between reference and test scenery across the field can then be calculated [32]. At first, the landmark was shifted into a distant area (roughly $100 \mathrm{~m}$ away). The picture comparisons revealed high image differences between the training and distant test field. Indeed, even in front of the landmark, a great part of the panoramic view is very different from that found at an equivalent position on the training field. Within the training area, removing the landmark does not significantly alter the view at the beginning of the route but results in high image differences near the nest position (Figure 2A). Indeed, the visual area covered by the landmark (or here, absence of landmark) is negligible at the feeder but increases as a tangent function as one approaches the nest (Figure 1, see also Additional file 1). Similarly, shifting the landmark by $16^{\circ}$ or $32^{\circ}$ does not significantly alter the view at the beginning of the route but creates high image differences at the real nest position.

The $16^{\circ}$ displacement creates a region of high image difference in the area opposite to the displaced landmark. This results in a valley of lower image differences between the feeder and the landmark. Within this valley, a zone of higher mismatch is located around 7-8 $\mathrm{m}$ on the way towards the $16^{\circ}$ displaced landmark (Figure $2 \mathrm{~B}$ ). The presence of this latter zone of mismatch is easily explained. As one moves from the feeder towards the displaced landmark, the image differences result from two competing factors: the landmark and the rest of the panorama. As a result of moving away from the training direction, the perceived distant panorama (i.e., all the scenery except the landmark) becomes more and more altered, thus steadily increasing the mismatch. The landmark, however, matches its target counterpart perfectly, and although very small at the beginning of the route (filling $<5 \%$ of the azimuth), it increases in size sharply with distance, thereby minimizing the global mismatch. 

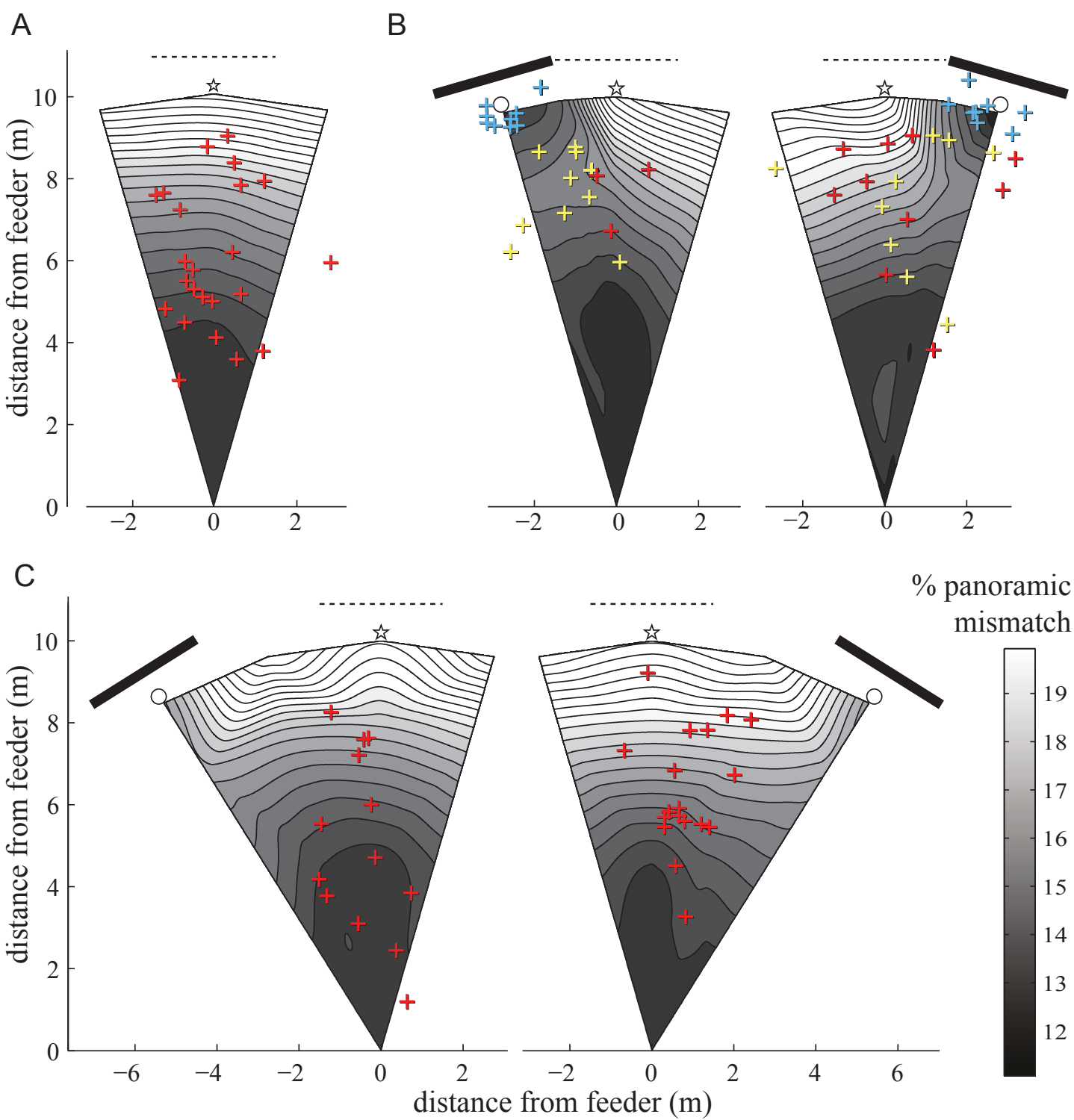

$\%$ panoramic mismatch

distance from feeder $(\mathrm{m})$

Figure 2 Maps of panoramic mismatches and first U-turn locations. The maps result from comparing panoramic pictures taken during Removal of the landmark (A), Rotation $16^{\circ}$ (B) and Rotation $32^{\circ}$ (C) tests with reference pictures from the training condition. "\% of panoramic mismatch' indicates the percentage of mismatching pixel across the image. Locations for comparisons are shown in the Additional file 1. Mismatch levels were then interpolated between those locations (triangle-based cubic interpolation). The darker the shade, the lower the mismatch between views. Each cross represents the location of the first U-turn (walking at least $20 \mathrm{~cm}$ back towards the feeder within $50 \mathrm{~cm}$ of displacement) of the ants. Red crosses: first U-turns of ants that never searched densely in front of the landmark. Yellow crosses: first U-turn of ants that displayed a U-turn before searching in front of the landmark. Blue crosses: first U-turn of ants that displayed no U-turn before searching in front of the landmark (Blue crosses thus correspond to the beginning of the search). Bar: landmark position during test. Dashed line: landmark position during training. Stars: nest position. White circle: fictive nest position relative to the landmark.

In combining landmark and panorama, the panoramic image differences grows as an ant travels from the feeder towards the landmark until a point of maximum mismatch (around 7-8 $\mathrm{m}$ along the feeder nest axis), beyond which the increasing size of the matching landmark diminishes the global mismatch (Figure 2B).

It is important to emphasise that the distributions of image differences presented here are not intended to model a particular homing strategy such as matching gradient descent, but simply allowed us to quantify the modification of the panoramic scenery the ants were subjected to during the tests. Whatever the actual process involved, any guidance strategy based on panoramic input should lead to disrupted behaviour if the global scenery is too much altered. Therefore, if ants are guided by panoramic views, they should not be able to 
reach the nest position in any of the tests conditions, as all of them present substantial panoramic image differences around the nest location. With $16^{\circ}$ displacements of the landmark, however, the ants may end up searching in front of the displaced landmark, but their approaching route should then be altered while crossing the hill of high mismatch located at around 7-8 m.

\section{Panoramic pictures: rotational image difference}

Panoramic images can be rotated until they produce the best matching to the reference image. Here, rotational IDFs (i.e., Image Difference as a Function of the rotation) presented often two distinct best choices for matching (Figure 3A). One choice (distant panorama choice) was generally obtained while facing the same direction as during training, because the distant panoramas of both images overlap well. The other choice (landmark choice) was generally obtained while facing roughly towards the displaced landmark, because the landmarks of both images are superimposed.

At the beginning of the route, the 'distant panorama choice' provides a better matching value than the 'landmark choice' (Figure 3B, C) because the landmark appears very small and the distant panorama covers most of the view. However, as one travels towards the nest, the apparent size of the landmark increases and the part of the field of view covered by the distant panorama decreases. Therefore, the 'landmark choice' matching quality grows and the 'distant panorama choice' decreases in importance. Ants travelling towards their nest in the training direction may thus suddenly switch in orientation when the image difference along the 'distant panorama choice' becomes too bad or when the 'landmark choice' direction becomes better. Such a switch towards the landmark direction does not imply that the ant is now attending to the landmark, but just that the panoramic image difference is lower while facing in that new direction. Ants may also 'hesitate' between the two directions when they provide equivalent matching quality, leading potentially to wiggling paths for that part of the route (see examples Figure 4G).

Interestingly, a side difference arises in the $16^{\circ}$ conditions. When the landmark is displaced to the left, facing the landmark becomes the best matching rotation at earlier locations than when the landmark is displaced to the right (Figure 3B). Some ants might thus continue to walk in the training direction longer when the landmark is displaced to the right.

Following purely the strategy of walking in the best matching direction should nonetheless lead the ant to the displaced landmark in the $16^{\circ}$ condition (Figure $3 \mathrm{~B}$ ) and to the nest or the landmark in the $32^{\circ}$ condition (Figure 3C). However, the actual image difference value of the selected direction might be important too. Ants might stop following the best matching direction if the image difference is considered too high (Figure 2 displays the distribution of the actual best matching values).

\section{Ant responses: control condition}

Ants homing from the feeder were captured just before reaching their nest in front of the landmark, and released again at the feeder location. When the landmark was left at its original position, the ants ran their route home again readily (Figure 4A) showing that they were guided by the perceived scenery and were not affected by potentially conflicting information provided by their path integrator. However, changing either the

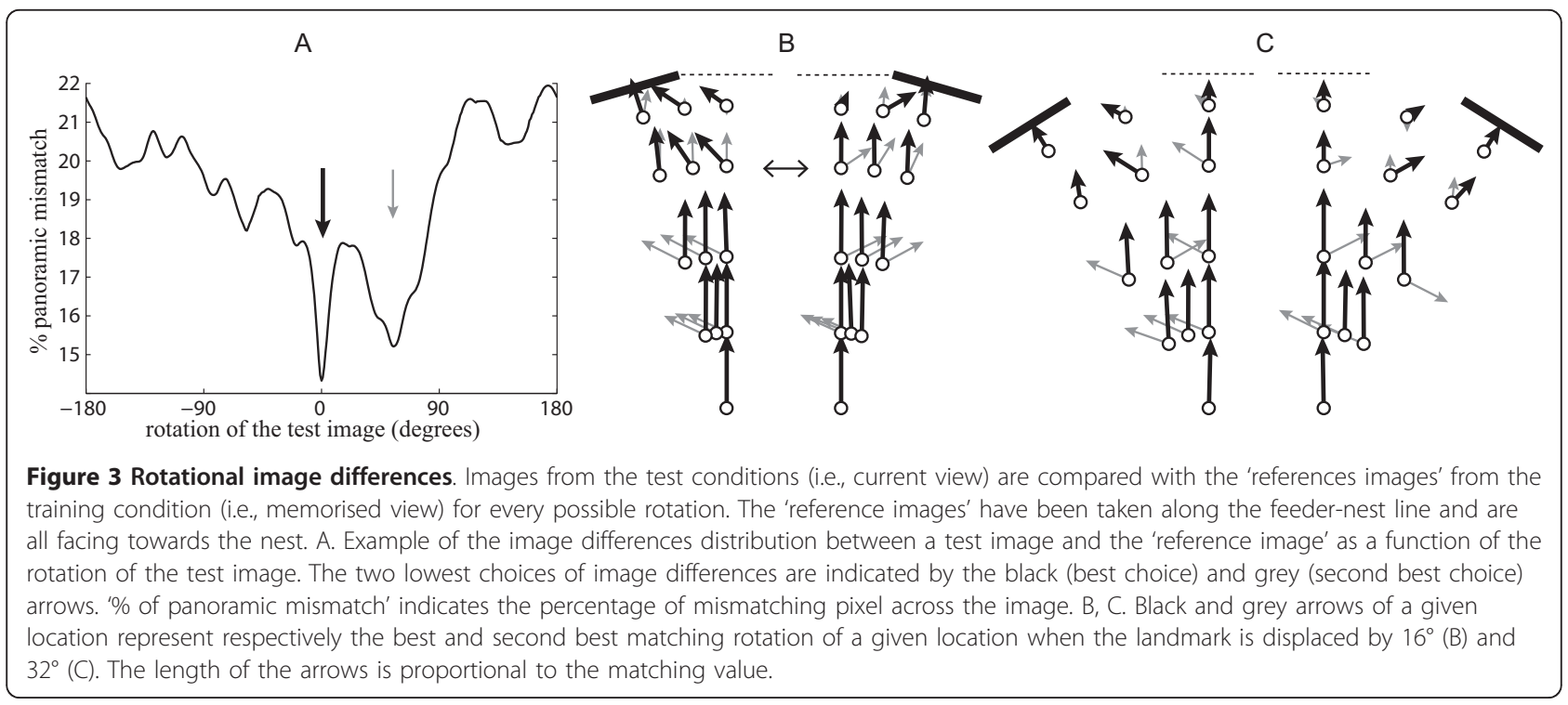




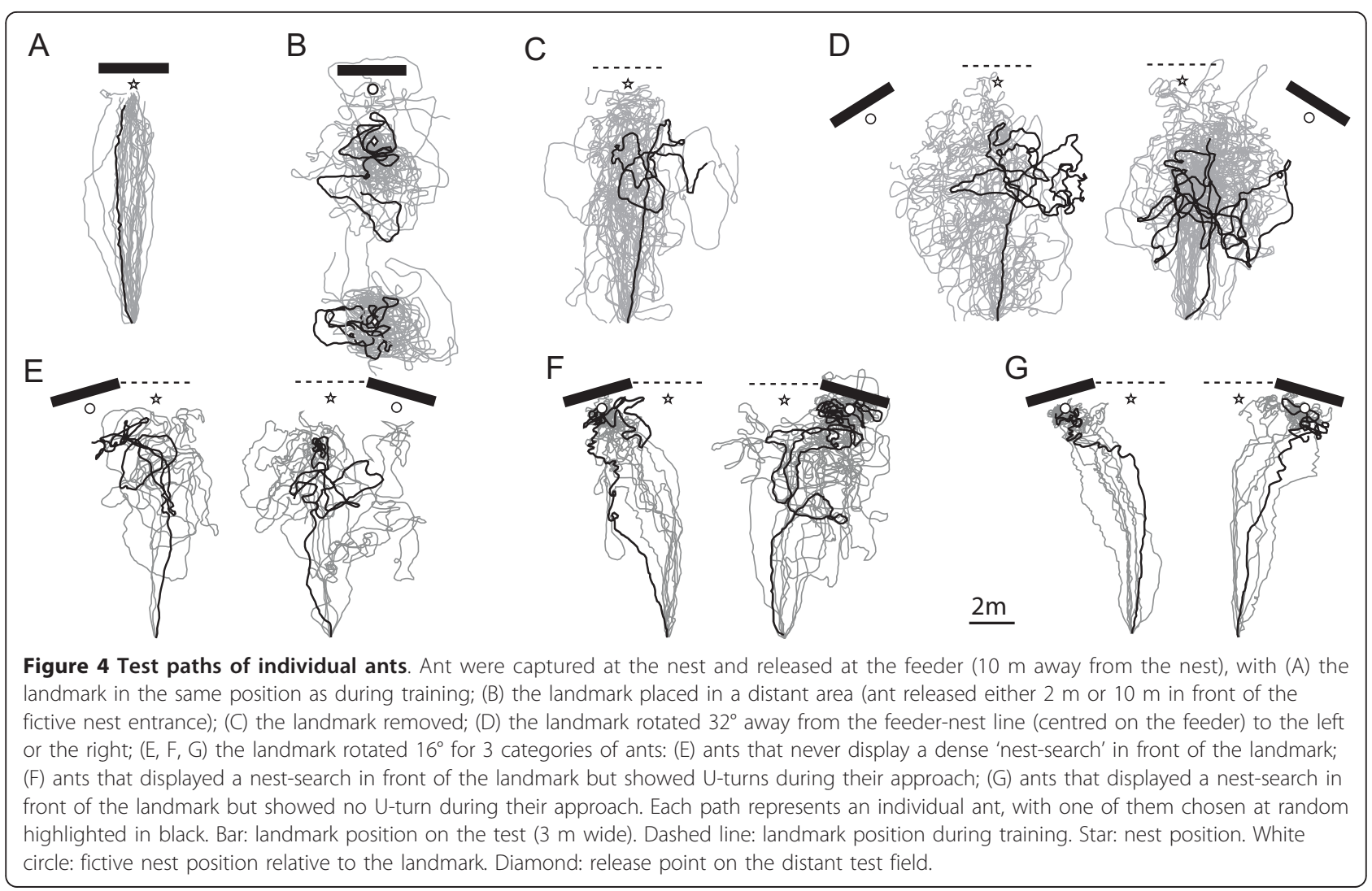

presence or the position of the landmark affected homing performance adversely, showing that the ants were affected by such alteration of the scenery.

\section{Ant responses: distant area, $32^{\circ}$ displacements and removal of the landmark}

When the landmark was translated to a distant area presenting an unfamiliar panorama, the ants released at 10 $\mathrm{m}$ (i.e., fictive feeder) or $2 \mathrm{~m}$ in front of the fictive nest engaged immediately in a search pattern at the release point and none of them ( 0 out of 16 and 0 out of 15 respectively) went searching at the fictive nest relative to the landmark (Figure 4B).

On the training field, with the landmark removed (Figure 4C) or displaced by $32^{\circ}$ (Figure 4D), the ants tended to run a first relatively straight segment but then displayed a U-turn on average half way from the nest and started searching. None of these ants (No-landmark: 0 out of 23; Left and Right $32^{\circ}$ displacements: 0 out of 31 ) found the nest or reached the fictive nest in front of the displaced landmark (within $3 \mathrm{~min}$ ). Interestingly, the approaches were on average centred along the feedernest axis in the no-landmark condition, but were a little bit skewed towards the displaced landmark in the $32^{\circ}$ condition (Additional file 2). The searches appeared centred on the first U-turn, but, interestingly, showed a larger spread than the searches displayed on the distant area (Additional file 2).

\section{Ant responses: $16^{\circ}$ displacements of the landmark}

With a smaller displacement of the landmark $\left(16^{\circ}\right)$, the ants displayed different behaviours, which we categorised into 3 groups (Figure 4E, F, G). Some ants (13 out 49) never reached the nest or searched for it in front of the displaced landmark (Figure 4E). The others (36 out of 49 individuals) eventually aimed at the landmark and displayed a dense search for the nest in front of it (Figure 4F, G). Determining whether (Figure 4F, G) or not (Figure 4E) an ant searched at the goal proved completely unambiguous, as two independent judges could agree completely: the 'nest-search' pattern would suddenly get much denser and the ants would not leave the area in front of the landmark for several minutes.

The 36 ants that searched for the nest (i.e., dense nestsearch) in front of the landmark were categorised in two groups depending on whether or not they displayed U-turns while approaching the landmark. U-turns consisted of more than a sharp turn, but also the stipulation that the ant walk back in a direction at least 113 degrees away from the training direction (i.e., went at least $20 \mathrm{~cm}$ down along the $\mathrm{Y}$ axis within $50 \mathrm{~cm}$ of travel). As a result, an ant could display very sharp turns to the left 
and to the right without being considered as displaying U-turns.

Around half of these ants (19 out of 36) displayed at least one U-turn before reaching the displaced landmark (Figure 4F), a much higher proportion than in the control group returning under training conditions (Fisher's exact test: $19 / 36$ vs. $1 / 25$, odds ratio $25.56, \mathrm{p}<0.0001$ ). Interestingly, their first U-turns were not located randomly along the route (Chi-square against random distribution across categories of $1 \mathrm{~m}: \chi^{2}=30, \mathrm{df}=9, \mathrm{p}<$ 0.0001 ) but occurred mostly between $7 \mathrm{~m}$ and $8 \mathrm{~m}$ away from the feeder (first U-turn average distance from the feeder \pm sd: $7.43 \pm 1.48 \mathrm{~m}$ ) (Figure $2 \mathrm{~B}$ yellow crosses, Figure 4F).

The other half (17 out of 36 ) approached the landmark without displaying any U-turns. The first U-turn of these 17 individuals occurred right in front of the landmark (Figure 2B blue crosses, Figure 4G) and, rather than showing uncertainty en route, corresponds to the beginning of the characteristic dense search for the nest entrance. However, a closer look at the approach of those individuals revealed an increasing tortuosity that reaches its maximum around 7 to $8 \mathrm{~m}$ away from the feeder, a pattern that was not observed in the control group (ANOVA groups*distances: $\mathrm{n}=17+25, \mathrm{~F}$ $=9.008, \mathrm{p}=0.0001$; between groups: $\mathrm{F}=12.971, \mathrm{p}=$ 0.0009) (Figure 5).

Overall, even though most ants searched for the nest in front of the $16^{\circ}$-displaced landmark, its displacement notably affected their approach. Their paths were more tortuous than in the control condition: wiggles and Uturns were strongest around 7 to $8 \mathrm{~m}$ away from the feeder.

\section{Path tortuosity and compass direction}

To test whether or not this degradation was due to the fact that the ants in the $16^{\circ}$ condition were led in a slightly different compass direction than during training, we focused on individuals that displayed long segments oriented towards the displaced landmark. Some ant paths (17 out of 48) presented a neat transition in the direction of travel, with a first segment oriented towards the nest and a second segment oriented towards the landmark (see Additional file 3 for examples). The switch in direction occurred on average around $5 \mathrm{~m}$ away from the feeder (average distance from the feeder $\pm \mathrm{sd}: 5.1 \pm 1.3 \mathrm{~m}$ ). Around half of those ants (8 out of 17) displayed a first U-turn while approaching the landmark. Those first U-turns did not occur immediately after the switch towards the landmark as it would be expected if the path disruption was due to the new compass direction of travel, but several meters thereafter (average distance between switch and first Uturn $\pm \mathrm{sd}$ : $3.8 \pm 0.9 \mathrm{~m}$ ), that is, around 7-8 $\mathrm{m}$ away from the feeder

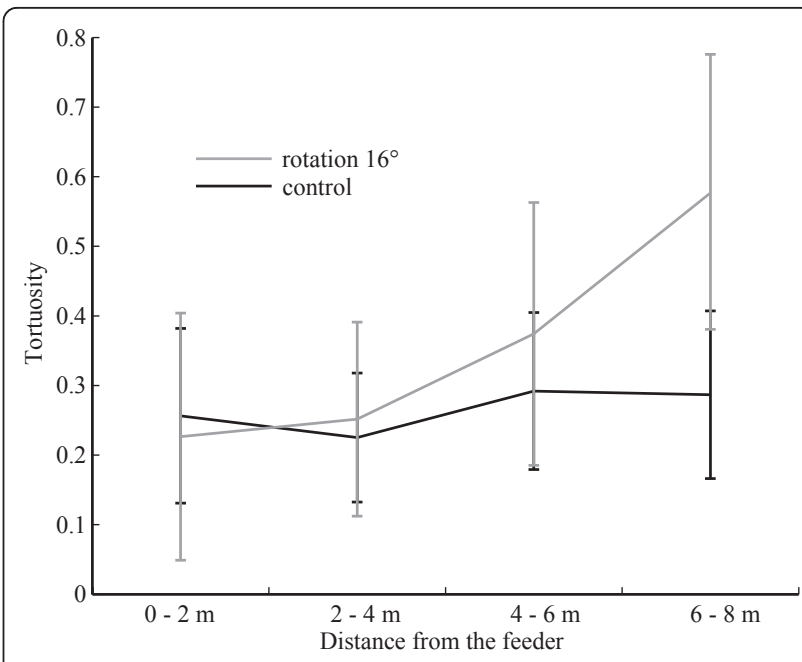

Figure $\mathbf{5}$ Tortuosity along the path. Index of path tortuosity at different distances away from the feeder $(\mathrm{M} \pm \mathrm{sem})$ for ants from the control group (in black) and from the Rotation $16^{\circ}$ condition (in grey) that displayed no U-turn before searching in front of the landmark. The tortuosity index corresponds to the averaged absolute angle (in radians) between the directions of successive chunks of $20-\mathrm{cm}$ line segments connecting points on the path. A circle of $20 \mathrm{~cm}$ radius was placed at the starting point of the digitised path, and where the circle intersected the path defined the first segment. The circle was then placed at the end of segment 1 to define segment 2, etc.

(average distance of the first U-turns from the feeder \pm sd: $7.9 \pm 1.1 \mathrm{~m})$.

Other ants (8 out of 48 ) headed towards the $16^{\circ}$-displaced landmark from the start (see Additional file 3 for examples). Although the direction of travel was similarly oriented towards the landmark all along their approach (heading direction: paired sample t test: 0-4 meter vs. 48 meter, $\mathrm{t}=-0.508, \mathrm{p}=0.627)$, the tortuosity of their paths increased significantly in the second half of the journey (tortuosity: paired sample t test: 0-4 meter vs. 48 meter, $\mathrm{t}=-4.635, \mathrm{p}=0.002)$ and half of them ( 4 out of 8) also displayed a first U-turn after $5 \mathrm{~m}$ of travel towards the landmark (first U-turn average distance from the feeder $\pm \mathrm{sd}$ : $6.5 \pm 1.3$ ).

Overall, ants were not equally perturbed everywhere along their way towards the landmark. Their paths were disrupted mostly around $7 \mathrm{~m}$ away from the feeder, independently of the ant's compass direction of travel. It seems therefore unlikely that the observed degradation of the path results from a discrepancy between the landmark direction and a memorised celestial compass information.

\section{Side differences}

In both $16^{\circ}$ and $32^{\circ}$ conditions, displacing the landmark to the left or to the right had different effects on the ants' first U-turn location. In $16^{\circ}$ conditions, U-turn 
location differences appeared along the $\mathrm{x}$-axis. When the landmark was displaced to the right, U-turns occurred closer to the feeder-nest axis and further away from the landmark side than when the landmark was displaced to the left ( $t$-test independent samples (values mirrored for one side): $\mathrm{t}=4.254, \mathrm{p}=0.0002$ ). Remarkably, such a difference was predicted by the panoramic image comparisons (see "rotational matching of panoramic views"). Along the $y$-axis, the distribution of first U-turns were similar on average ( $\mathrm{t}$-test independent samples: $\mathrm{t}=$ $1.587, \mathrm{p}=0.1234$ ) but were more spread in the $16^{\circ}$ Right condition (Levene's test: $\mathrm{F}=6.376, \mathrm{p}=0.0170$ ).

With $32^{\circ}$ displacement of the landmark, no side differences in U-turns distribution appeared along the $\mathrm{x}$-axis ( $\mathrm{t}$-test independent samples: $\mathrm{t}=-1.208, \mathrm{p}=0.2368$ ). Along the y-axis, however, U-turns occurred significantly earlier ( $t$-test independent samples: $t=14.047$, $\mathrm{p}<0.0001)$ and were significantly more scattered (Levene's test: $\mathrm{F}=6.128, \mathrm{p}=0.0190$ ) when the landmark was displaced to the right.

\section{Discussion}

Melophorus bagoti lives a habitat full of landmark information such as bush, trees or distant cliffs, and evolution has tuned those ants to learn quickly [36] and rely heavily on the so called 'landmark information' [7]. We here investigate whether navigating ants functionally segregate the perceived scenery into landmarks for guidance and the panorama as contextual cue. Such theories infer that, for the animal, the given landmark is somewhat isolated from the rest of the panorama. For this purpose, we gave ants every incentive to isolate a landmark from the panorama by choosing an area devoid of proximal trees, by clearing that area of any proximal clutter and providing them with a particularly prominent artificial landmark at the nest entrance.

\section{Initial segments and searches}

The ants accustomed to the landmark behind their nest were captured as zero-vector ants (i.e., just before reaching their nest entrance) and released again at the feeder position. Because the 'zero state' of their path integrator cannot provide them with a homing direction, zero-vector ants have to rely on the visual surroundings to home. In a landmark rich habitat, the recognition of the surrounding overrides completely the information given by the path integrator $[7,9,37]$. It is therefore not surprising that when the landmark was left at its original position, the recognition of familiar surroundings led zero-vector M.bagoti ants to run their home route again readily and thoroughly (Figure $4 \mathrm{~A}$ ).

When the landmark was displaced from the training position, however, the ant routes were notably altered, revealing that such modification of the scenery affected their homing. When released on the distant test field, the large landmark was not used: ants engaged immediately in a systematic search around the release point (Figure 4B) as they typically do when released in an unfamiliar environment [38]. When the landmark was removed from the training field or displaced by $16^{\circ}$ or $32^{\circ}$ to the sides, most of the ants ran first a relatively straight segment, showing that they recognised the scenery at the beginning of the route (Figure 4C, D, E, F, G). Whether the ants recalled a local vector (i.e., segment of travel based on compass information) or used a view based matching strategy to achieve this first segment cannot be properly disentangled here. But previous work on this species showed that panorama can be matched and used independently of the compass direction [26] stressing the use of a view based matching strategy rather than a local vector. Moreover, some approaches were here skewed towards the landmark in both $16^{\circ}$ and $32^{\circ}$ conditions (see Additional file 2), contesting the hypothesis of a pure local vector.

The ants from the $32^{\circ}$ displacements (Figure 4D) or no-landmark conditions (Figure $4 \mathrm{C}$ ) engaged in winding search loops on average half-way to the nest. None searched at the real nest or at the fictive nest position in front of the landmark. Interestingly, these searches were more spread than the systematic search displayed on unfamiliar terrain, revealing that other factors, possibly view based matching or compass information, were influencing the search pattern (Additional file 2).

\section{Guidance is not focused on the landmark}

The hypothesis assuming that the distant panorama is not used for guidance but as a contextual cue provides an explanation for the behaviours described above. The panoramic context could be seen as delivering a negative verdict, rendering the landmark not worth approaching, and triggering the observed search behaviours. However, two pieces of evidence show that guidance was not purely based on the landmark, and that ants were attending simultaneously to other cues from the panorama.

Firstly, displacing the landmark to the left or to the right had different effects on the ants. A $16^{\circ}$ displacement to the left led the ants to meander more towards the landmark and less towards the feeder-nest middle line than a $16^{\circ}$ displacement to the right. And a $32^{\circ}$ displacement to the left had an earlier impact on the ants' paths than a $32^{\circ}$ displacement to the right. Since the landmark presented highly contrasted edges against the background, such side differences should not have arisen if guidance was purely based on the landmark.

Secondly, in the $16^{\circ}$ displacement condition, some ants ended up searching for their nest in front of the landmark, but their approach did not resemble the 
straight approach found in control ants (compare Figure 4A with Figures 4F, G). Instead, they exhibited behaviours indicative of 'uncertainty': they U-turned or showed more tortuous paths during their approach (Figure 4F, G). The presence of this uncertainty in the ant paths was independent of their direction of travel, rejecting the hypothesis that uncertainty was resulting purely from a conflict with a stored local vector (i.e., a memorised compass direction) pointing along the training direction. Consistent with this, previous work showed that M.bagoti can readily match and use familiar panorama presented in a wrong compass direction [26]. As ants reached - and therefore used - the displaced landmark, the path uncertainty observed cannot be attributed to a negative verdict of a hypothetical contextual cue either. Such path uncertainty must therefore result from an alteration of the terrestrial cues the ants were using for guidance. As the highly contrasted landmark was not altered in itself, we can conclude that guidance was simultaneously based on other terrestrial cues.

\section{Functional segregation landmarks/panorama or panoramic views?}

As guidance was not focused on the landmark only, the class of theories assuming a functional segregation between panorama (as context) and landmarks (as guidance cues) needs to invoke other processes like the simultaneous use of other landmarks extracted from the distant landscape for guidance and not for context. But then the process of deciding which landmarks are to be used for guidance and which ones are used as contextual cues appears complex and cannot be based on a simplistic distinction between proximal landmarks and distal panorama. We find it most parsimonious to account for the results by proposing that the ants in our experiment were using guidance strategies based on large panoramic views, without summoning the need to segregate such panoramic views into context and landmarks.

By comparing panoramic images in simple ways, we could explain here the sharp transitions in the direction of travel observed, the presence of wiggling paths at particular locations, some of the differences observed when displacing the landmark to the left or to right (see 'Rotational matching of panoramic views' in Results), as well as why the ant routes were disrupted at different locations across groups (U-turns and searching) (see 'Panoramic image difference distribution' in Results). The correspondence between the regions where the ants' travel was disrupted and the regions of high panoramic image differences (on average $\sim 15 \%$ in this case, but with individual variation) (Figure 6) suggests that guidance cues must be widespread on the ants' panoramic visual field.

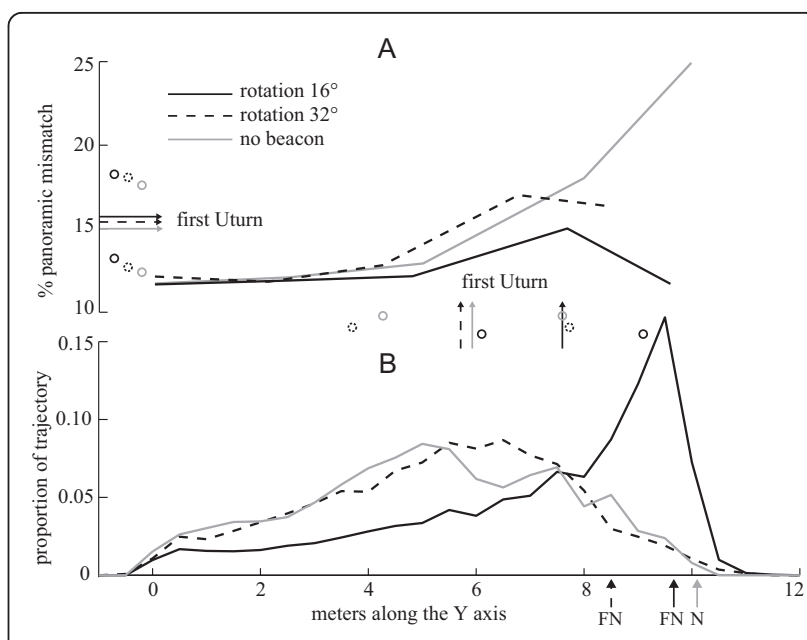

Figure 6 Panoramic mismatches and search distribution along the Y-axis. A. Panoramic mismatch along the feeder-landmark line for the Rotation $16^{\circ}$ and $32^{\circ}$ tests and along the feeder-nest line for the no-landmark test. The vertical arrows represent the average position of the first U-turn displayed by each ant (counting only ants that displayed U-turns before reaching the landmark) (ANOVA between groups: $F=11.096 ; p<0.0001$; all pairs Tukey's post hoc: Rotation $16^{\circ} \mathrm{a}$, Rotation $32^{\circ} \mathrm{b}$, no-landmark b). The horizontal arrows represent the average value of mismatch where the first U-turns were displayed (inferred from the 2D distribution of image difference, Figure 2) (ANOVA between groups: $F=0.3823 ; p=$ 0.6835). The open circles on the side of each arrow indicate the inter-individual standard deviation. B. Search distribution of the ants along the Y-axis. Paths were limited to the first $30 \mathrm{~m}$ travelled. The relative distribution was first calculated for each individual and then pooled for each condition so that each individual ant contributed equally to the distribution. The arrows indicate the position of the nest (for the no-landmark condition) or the fictive nest in front of the landmark (for Rotation $16^{\circ}$ and $32^{\circ}$ conditions).

\section{How to match panoramic views?}

Despite a great amount of work [31,32,35,39-42] how insects match memorised and current views to produce such efficient navigational behaviours is far from fully understood. Recent evidence shows that ants are able to align their body in order to match the retinal position of the features memorized along a familiar route [40]. Such a simple mechanism based on panoramic images also explains spontaneous biases in ant routes observed in an artificial arena [31].

The present work also supports this hypothesis for route following (see 'Rotational matching of panoramic views' in Results), but suggests that ants may not always follow that strategy. Indeed, in the $32^{\circ}$ condition, the ants U-turned and started searching on average half-way to the nest although our analysis of rotational image differences shows that walking in the best matching directions should lead the ants all the way towards the nest or the landmark. We suggest that individual ants may possess a mismatch tolerance threshold that allows them to switch between navigational strategies. If the 
mismatch is considered too bad, ants might stop such a route strategy (i.e., walking along the best matching direction) and start another strategy. Due to the artificial alteration of the scenery in this situation, this second strategy led the ants to meander in loops, but in a different fashion than the systematic search displayed in a totally unknown area (see Additional file 2). In more naturalistic situations, such a strategy might be adapted for navigation in less familiar environments that do not show excessive mismatch, such as situations in which the ant has been led astray or blown away from her familiar route corridor by a small distance (work in preparation).

Although analysing panoramic images explains a lot, other puzzles remained. We did not manage to explain the early U-turns observed in the $32^{\circ}$ left condition. A better knowledge of the nature of insects' perceptions and memories would be precious for further illuminating guidance mechanisms.

\section{Nature of the insect views?}

Do insects use landmarks for guidance? Yes. Much evidence in bees, wasps and ants shows that insects are highly influenced by landmarks (for a review [18]; in Melophorus bagoti $[9,13])$. Do insects focus on individual landmarks only, filtering out the distant panorama from guidance mechanisms? We think not. The present work shows that, even when the dichotomy between proximal landmark and distal panorama is artificially emphasised, guidance is not focused solely on the landmark. But then, are insects' panoramic views constituted of an ensemble of individual landmarks? Probably not. Evidence shows that insects store a pallet of features/ parameters like strong boundaries [43], spots of light, centre of gravity, and colour of areas [44] and appear to do so without reconstructing the actual pattern [45]. Insects also have access to landmark distance information based on motion parallax [20-25]. The motion parallax creates a pattern of optic flow that can be used to pinpoint a target location [21]. As with static cues, such dynamic cues can potentially be matched across the whole panoramic view [46] and the insect may not be using them exclusively on isolated landmarks. Rather than isolated landmarks, encoding such a pallet of static and dynamic parameters simultaneously across a large part of the retina seems an appropriate strategy to cope with the complexity of natural scenes and the poor resolution of insects' eyes.

\section{Conclusion}

We have created conditions in which a landmark seemed prominent, easy to extract, and very useful, at least to our primate visual system. But we (primates) have high acuity frontal foveal vision that can be focused on individual objects. Added to that is an entire specialised stream, the so-called ventral stream that is dedicated to object perception $[47,48]$. To those humans who have seen this landmark, it seemed the obvious one to use. Yet the evidence suggests that the ants did not focus only on the landmark but relied simultaneously on the distant panorama for guidance. Is this pattern peculiar to our experimental situation? We have reasons to think that the use of panoramas as a whole would be more widespread in insect navigation. Using cues that are encoded and processed simultaneously across a large part of the retina can well explain present and past results obtained in ants and seems an appropriate strategy to cope with the complexity of natural scenes, the poor resolution of insects' eyes, and the lack of dedicated object-perception visual streams. It is still unclear what the nature of the parameters is that comprise insects' perceptions and memories, but future studies should not assume that insects functionally segregate landmarks and distal panorama without evidence for such a dichotomy.

\section{Methods}

\section{Nest area and Landmark}

We chose a nest located in an area devoid of any proximal trees and provided the ants with a feeder $10 \mathrm{~m}$ away from their nest. The area between the nest and feeder, where the ants navigated, was open, flat, and cleared of any natural debris. The artificial landmark consisted of a huge black sheet $(3 \mathrm{~m}$ wide and $2 \mathrm{~m}$ high) stretched between two poles $90 \mathrm{~cm}$ behind the nest entrance (Figure 1). The landmark width subtended an angular size of $118^{\circ}$ at the nest location and $15^{\circ}$ at the most distant location (i.e., feeder). Melophorus bagoti acuity being about $4^{\circ}$ [49], the landmark could be perceived all along their homeward route. To the ants, the landmark presented a strong dynamic change in size (increasing in retinal angle of $64^{\circ}$ (from $54^{\circ}$ to $118^{\circ}$ ) along the azimuth in the last $2 \mathrm{~m}$ of the route). In contrast, the rest of the panorama presented very little apparent displacement, the closest tree being located roughly $14 \mathrm{~m}$ away behind the nest. All in all, our artificial landmark stood as an obvious beacon for the nest entrance.

\section{Protocol}

$M$. bagoti lives in the semi-arid terrain of central Australia, which is typically filled with bushes, grass tussocks and trees. The ants were given food ad libitum in a fixed feeder $10 \mathrm{~m}$ from the nest entrance, and painted at their first visit to the feeder with a colour that marked the day of arrival. After 2 days of spontaneous shuttling between the nest and the feeder, with the artificial landmark immediately behind the nest, the marked 
foragers were tested. An ant returning from the feeder was captured near the nest and released again at the feeder location with the artificial landmark either left at its original position, removed or displaced by $0^{\circ}, \pm 16^{\circ}$, or \pm $32^{\circ}$ relative to the feeder-nest direction. Another test consisted of releasing the ants $10 \mathrm{~m}$ or $2 \mathrm{~m}$ in front of an identical landmark located in a distant test field roughly $100 \mathrm{~m}$ away in the same absolute orientation as in the training condition. Ants were tested singly, and each ant was only tested once.

\section{Path analysis}

The training and test fields were covered by a grid of 1$\mathrm{m}$ squares made out of strings stretched between tent pegs that allowed the recording of paths by hand. The recorded paths were digitised into $(x, y)$ coordinates with the software Graphclick ${ }^{\mathrm{TM}}$ http://www.arizona-software.ch/ and processed using Matlab ${ }^{\mathrm{TM}}$ (Math Works, Natick, MA, USA) programs. Paths were analysed for Uturns and tortuosity. U-turns were defined as walking back at least $20 \mathrm{~cm}$ along the $\mathrm{Y}$ axis within $50 \mathrm{~cm}$ of displacement. The maximum angle away from the training direction that can be travelled for more than $50 \mathrm{~cm}$ without being considered as U-turn was thus 113 degrees. The tortuosity index of a path corresponded to the averaged absolute turn angle (in radians) between successive chunks of $20-\mathrm{cm}$ line segments connecting points on the path. A circle of $20 \mathrm{~cm}$ radius was placed at the starting point of the digitised path, and where the circle intersected the path defined the first segment. The circle was then placed at the end of segment 1 to define segment 2, etc.

\section{Panoramic images analysis}

To quantify changes in the visual panorama generated by displacing the artificial landmark, 5 reference panoramic pictures (black and white $360 * 40$ pixels) were taken along the trained route, from the feeder to the nest, with the landmark in the training position. For each test condition, with the landmark displaced or removed, we mapped the area explored by the ants with 17 pictures (see Additional file 1). The test pictures were compared to the reference picture that corresponded to the same distance from the feeder. This was always the reference picture that best matched the tested picture. To compare each image to a reference image, we calculated the pixel-wise RMS (route mean square) error for all possible orientations of the reference image. The pixel-wise RMS gives us a value for the mismatch, or image difference, between two images. The RMS of the best matching orientation was recorded for each of the 17 tests pictures and used for the construction of the image difference map (see Figure 2). Interpolation of image differences values using the 17 pictures provided an estimate of mismatch across the whole terrain of travel.

\section{Additional material}

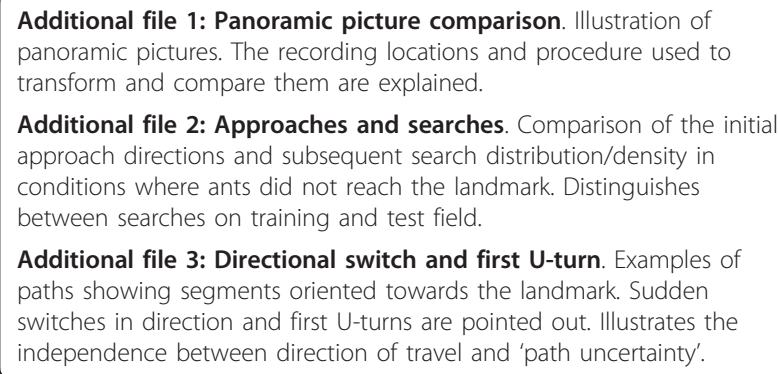

Additional file 3: Directional switch and first U-turn. Examples of paths showing segments oriented towards the landmark. Sudden switches in direction and first U-turns are pointed out. Illustrates the independence between direction of travel and 'path uncertainty'.

\section{Acknowledgements}

We thank Sebastian Schwarz, Patrick Schultheiss and Madeleine Tempé for their help on the field, and Paul Graham for helping, lending his panoramic camera, and providing comments on the manuscript. We also wanted to thank two reviewers for many thoughtful remarks and suggestions. This research was supported by a Macquarie University research excellence scholarship (iMQRES) to AW and an Australian Research Council Discovery Project grant (DP110100608). We thank the CSIRO Centre for Arid Zone Research for letting us use their grounds for research.

\section{Author details}

'Department of Biological Sciences, Macquarie University, Sydney, NSW 2109 Australia. ${ }^{2}$ Centre de Recherches sur la Cognition Animale, CNRS, UMR 5169, Université Paul-Sabatier, Toulouse F-31062, France.

\section{Authors' contributions}

AW, GB and KC designed the experiment. AW carried out the experiment, performed the analysis and wrote the manuscript. All authors read, revised, and approved the final manuscript.

\section{Competing interests}

The authors declare that they have no competing interests.

Received: 13 May 2011 Accepted: 27 August 2011

Published: 27 August 2011

\section{References}

1. Cartwright BA, Collett TS: Landmark Learning in Bees-Experiments and Models. J Comp Physiol A-Neuroethol Sens Neural Behav Physiol 1983, 151:521-543.

2. Durier V, Graham P, Collett TS: Snapshot memories and landmark guidance in wood ants. Curr Biol 2003, 13:1614-1618.

3. Tinbergen N, Kruyt W: Über die Orientierung des Bienenwolfes (Philanthus triangulum Fabr.). Z vergl Physiol 1938, 25:292-334.

4. Wehner R, Räber F: Visual spatial memory in desert ants, Cataglyphis bicolor (Hymenoptera: Formicidae). Experientia 1979, 35:1569-1571.

5. Collett TS: Landmark learning and guidance in insects. Philos Trans $R$ Soc Lond Ser B-Biol Sci 1992, 337:295-303.

6. Collett TS: Insect navigation en route to the goal: Multiple strategies for the use of landmarks. J Exp Biol 1996, 199:227-235.

7. Kohler M, Wehner R: Idiosyncratic route-based memories in desert ants, Melophorus bagoti: How do they interact with path-integration vectors? Neurobiol Learn Mem 2005, 83:1-12.

8. Macquart D, Garnier L, Combe M, Beugnon G: Ant navigation en route to the goal: signature routes facilitate way-finding of Gigantiops destructor. J Comp Physiol A-Neuroethol Sens Neural Behav Physiol 2006, 192:221-234.

9. Wystrach A, Schwarz S, Schultheiss P, Beugnon G, Cheng K: Views, landmarks, and routes: how do desert ants negotiate an obstacle 
course? J Comp Physiol A-Neuroethol Sens Neural Behav Physiol 2011, 197:167-179.

10. Åkesson S, Wehner R: Visual navigation in desert ants Cataglyphis fortis: are snapshots coupled to a celestial system of reference? J Exp Biol 2002, 205:1971-1978.

11. Judd SPD, Collett TS: Multiple stored views and landmark guidance in ants. Nature 1998, 392:710-714

12. Narendra A: Homing strategies of the Australian desert ant Melophorus bagoti - II. Interaction of the path integrator with visual cue information. J Exp Biol 2007, 210:1804-1812.

13. Narendra A, Si A, Sulikowski D, Cheng K: Learning, retention and coding of nest-associated visual cues by the Australian desert ant, Melophorus bagoti. Behav Ecol Sociobiol 2007, 61:1543-1553.

14. Graham P, Fauria K, Collett TS: The influence of beacon-aiming on the routes of wood ants. J Exp Biol 2003, 206:535-541.

15. Collett TS, Kelber A: The Retrieval of Visuo-Spatial Memories by Honeybees. J Comp Physiol A-Sens Neural Behav Physiol 1988, 163:145-150.

16. Cheng K: Arthropod navigation: ants, bees, crabs, spiders finding their way. In Comparative cognition: experimental explorations of animal intelligence. Edited by: Wasserman EA, Zentall TR. Oxford: Oxford University Press; 2006:

17. Collett TS, Collett M: Memory use in insect visual navigation. Nat Rev Neurosci 2002, 3:542-552

18. Collett TS, Graham P, Harris RA, Hempel-De-lbarra N: Navigational memories in ants and bees: Memory retrieval when selecting and following routes. Advances in the Study of Behavior 2006, 36:123-172.

19. Cartwright BA, Collett T: Landmark maps for honeybees. Biol Cybern 1987, 57:85-93.

20. Cheng K, Collett TS, Pickhard A, Wehner R: The use of visual landmarks by honeybees-bees weight landmarks according to their distance from the goal. J Comp Physiol A-Sens Neural Behav Physiol 1987, 161:469-475.

21. Dittmar L, Sturzl W, Baird E, Boeddeker N, Egelhaaf M: Goal seeking in honeybees: matching of optic flow snapshots? J Exp Biol 2010, 213:2913-2923.

22. Lehrer M, Collett TS: Approaching and departing bees learn different cues to the distance of a landmark. J Comp Physiol A-Sens Neural Behav Physiol 1994, 175:171-177.

23. Lehrer M, Srinivasan MV, Zhang SW, Horridge GA: Motion cues provide the bees visual world with a 3rd dimention. Nature 1988, 332:356-357.

24. Sobel EC: The locust's use of motion parallax to measure distance. J Comp Physiol A-Sens Neural Behav Physiol 1990, 167:579-588

25. Zeil J: Orientation flights of solitary wasps (Cerceris, Sphecidae, Hymenoptera) 2. Similarities between orientation and return flights and the use of motion parallax. J Comp Physiol A-Sens Neural Behav Physiol 1993, 172:207-222.

26. Graham $P$, Cheng $K$ : Ants use the panoramic skyline as a visual cue during navigation. Curr Biol 2009, 19:R935-R937.

27. Reid SF, Narendra A, Hemmi JM, Zeil J: Polarised skylight and the landmark panorama provide night-active bull ants with compass information during route following. J Exp Biol 2011, 214:363-370.

28. Wehner $R$, Müller M: Piloting in desert ants: pinpointing the goal by discrete landmarks. J Exp Biol 2010, 213:4174-4179.

29. Wystrach $A$ : Ants in rectangular arenas: $A$ support for the global matching theory. Commun Integr Biol 2009, 2:388-390.

30. Wystrach A, Beugnon G: Ants Learn Geometry and Features. Curr Biol 2009, 19:61-66.

31. Wystrach A, Sosa S, Cheng K, Beugnon G: Geometry, features and panoramic views: ants in rectangular arenas. J Exp Psychol Anim Behav Process

32. Zeil J, Hofmann MI, Chahl JS: Catchment areas of panoramic snapshots in outdoor scenes. J Opt Soc Am A 2003, 20:450-469.

33. Pastergue-Ruiz I, Beugnon G, Lachaud JP: Can the ant Cataglyphis cursor (Hymenoptera, Formicidae) encode global landmark-landmark relationships in addition to isolated landmark-goal relationships. J Insect Behav 1995, 8:115-132.

34. Graham P, Durier V, Collett TS: The binding and recall of snapshot memories in wood ants (Formica rufa L.). J Exp Biol 2004, 207:393-398.

35. Stürzl W, Cheung A, Cheng K, Zeil J: The information content of panoramic images I: The rotational errors and the similarity of views in rectangular experimental arenas. J Exp Psychol Anim Behav Process 2008, $34: 1-14$
36. Schwarz $\mathrm{S}$, Cheng $\mathrm{K}$ : Visual associative learning in two desert ant species. Behav Ecol Sociobiol 2010, 64:2033-2041.

37. Andel D, Wehner R: Path integration in desert ants, Cataglyphis: how to make a homing ant run away from home. Proc $R$ Soc Lond Ser B-Biol Sci 2004, 271:1485-1489.

38. Schultheiss $P$, Cheng $K$ : Finding the nest: inbound searching behaviour in the Australian desert ant, Melophorus bagoti. Anim Behav 2011, 81:1031-1038.

39. Collett M: How desert ants use a visual landmark for guidance along a habitual route. Proc Natl Acad Sci USA 2010, 107:11638-11643.

40. Lent DD, Graham P, Collett TS: Image-matching during ant navigation occurs through saccade-like body turns controlled by learned visual features. Proc Natl Acad Sci USA 2010, 107:16348-16353.

41. Möller R, Maris M, Lambrinos D: A neural model of landmark navigation in insects. Neurocomputing 1999, 26-7:801-808.

42. Möller R, Vardy A: Local visual homing by matched-filter descent in image distances. Biol Cybern 2006, 95:413-430.

43. Harris RA, Graham P, Collett TS: Visual cues for the retrieval of landmark memories by navigating wood ants. Curr Biol 2007, 17:93-102.

44. Ernst $\mathrm{R}$, Heisenberg M: The memory template in Drosophila pattern vision at the flight simulator. Vision Res 1999, 39:3920-3933.

45. Horridge GA: Recognition of a familiar place by the honeybee (Apis mellifera). J Comp Physiol A-Neuroethol Sens Neural Behav Physiol 2005 191:301-316

46. Dittmar L: Static and dynamic snapshots for goal localization in insects? Commun Integr Biol 2011, 4:17-20.

47. Goodale MA, Milner AD: Separate visual pathways for perception and action. Trends Neurosci 1992, 15:20-25.

48. Mishkin M, Ungerleider L, Macko K: Object vision and spatial vision: two cortical pathways. Trends Neurosci 1983, 6:414-417.

49. Schwarz S, Narendra A, Zeil J: The properties of the visual system in the Australian desert ant Melophorus bagoti. Arthropod Structure \& Development 2011, 40:128-134.

doi:10.1186/1742-9994-8-21

Cite this article as: Wystrach et al:: Landmarks or panoramas: what do navigating ants attend to for guidance? Frontiers in Zoology 2011 8:21.

\section{Submit your next manuscript to BioMed Central and take full advantage of:}

- Convenient online submission

- Thorough peer review

- No space constraints or color figure charges

- Immediate publication on acceptance

- Inclusion in PubMed, CAS, Scopus and Google Scholar

- Research which is freely available for redistribution 